\title{
Surgical and oncological implications of aberrant arterial anatomy in patients undergoing pancreatoduodenectomy
}

Muhammad Rizwan Khan

Saleema Begum

Dawar Burhan Khan

K. M. Inam Pal

Follow this and additional works at: https://ecommons.aku.edu/pakistan_fhs_mc_surg_gen

Part of the Oncology Commons, Radiology Commons, and the Surgery Commons 


\section{Surgical and oncological implications of aberrant arterial anatomy in patients undergoing pancreatoduodenectomy}

Muhammad Rizwan Khan1, Saleema Begum², Dawar Burhan Khan³, Khuwaja Mohammad Inam Pal4

\begin{abstract}
Whipple's pancreatoduodenectomy is a complex surgical procedure and any aberrant arterial anatomy may have serious surgical implications. The objective of our study was to analyse the frequency of aberrant hepatic artery and compare the outcomes in patients with normal anatomy. Clinical data and computed tomography scans of 45 consecutive patients who underwent Whipple's procedure from 2007 to 2016 were reviewed. Group 'A' included patients with aberrant hepatic artery while group 'B' with normal anatomy. Aberrant hepatic artery was present in 11 (24\%) patients and type $V$ was the most common variant $(n=5,45 \%)$. Morbidity rate in group $A$ was $82 \%$ and group B was $62 \%(p=0.288)$, while 30 -day mortality rate was $18 \%$ and $9 \%$ respectively $(p=0.582)$. There was no difference in the oncological clearance in both the groups. Aberrant hepatic artery does not seem to influence the morbidity, mortality and tumour resection margins in patients undergoing Whipple's procedure.
\end{abstract}

Keywords: Aberrant hepatic artery, pancreatoduodenectomy, accessory hepatic artery.

https://doi.org/10.5455/JPMA.4875

\section{Introduction}

The incidence of hepatic arterial anomalies has been reported from $20 \%$ to $40 \%$ in patients who underwent pancreatoduodenectomy (PD), which adds to the difficulty of an already technically challenging procedure. ${ }^{1}$ The variation in the hepatic artery anatomy was first described by Michels 2 in 1966, which was further updated by Hiatt et al in 1994. ${ }^{3}$ The most frequent arterial variant is the aberrant right hepatic artery ( $\mathrm{RHA}$ ) arising from the superior mesenteric artery (SMA), followed by aberrant left hepatic artery originated from left gastric artery. ${ }^{4}$ Replaced or accessory right hepatic artery from SMA is of great concern during pancreatoduodenectomy because the anomalous vessel can course near or through 1,2,4Department of Surgery, Aga Khan University, Karachi, Pakistan; 3Department of Radiology, Aga Khan University, Karachi, Pakistan Correspondence: Muhammad Rizwan Khan.e-mail: doctormrkhan@yahoo.com pancreatic head and posterior to common bile duct. 5 Similarly, a less common variant in which common hepatic artery arises from superior mesenteric artery can also impede dissection of pancreatic head, common bile duct and gastroduodenal artery (GDA) during PD.

The presence of accessory or replaced RHA from SMA may have serious surgical implications. RHA from SMA has high chances of being encompassed by cephalo-pancreatic tumours. ${ }^{6}$ During surgery hepatic artery arising from SMA may be inadvertently injured, leading to bile duct ischaemia resulting in compromised biliary enteric anastomosis since the major supply of common bile duct is from RHA. On the other hand, an attempt to preserve such arterial supply may compromise the R0 resection of the tumour leading to a positive margin of resection with negative influence on the oncological outcomes. Thus, presence of such anomalous anatomy may prolong the operative time and increase postoperative morbidity and mortality.

Aberrant arterial anatomy can be picked up on preoperative scans, however, the sensitivity and specificity of vascular involvement in pancreatic tumours is $60 \%$ and $94 \%$ only. ${ }^{7}$ The surgical technique may be planned beforehand but the definitive procedure may only be executed at the time of the surgical procedure.

The objective of our study was to analyze the frequency of anatomical variants of hepatic artery in patients who underwent PD at our hospital and to assess the surgical and oncological implications by comparing postoperative morbidity and mortality with those who had normal anatomy.

\section{Methods}

We conducted a retrospective review of the clinical data and computerized tomography scans (CT) of patients who underwent PD at Aga Khan University Hospital, Karachi, Pakistan from January 2007 to December 2016 after taking approval from institutional ethical review committee. Data collection was carried out in the month of November and 
December 2017. All preoperative CT scans were reviewed by a consultant radiologist for any variation in hepatic artery anatomy and classified according to Hiatt's classification. 3 Patients were divided into two groups depending upon the arterial anatomy. Group 'A' included patients who had anomalous arterial anatomy and group $B$ included patients with normal anatomy based on the preoperative $\mathrm{CT}$ findings.

The data were collected on several variables of clinical significance. The preoperative variables included age, gender and ASA level according to American society of Anaesthesiologists. Intraoperative variables like operating time, estimated blood loss, number of red blood packed cell transfusions and intraoperative detections of RHA arising from SMA. Postoperative variables included length of hospital stay, morbidity according to Clavien-Dindo classification, 8 mortality and resection type. Resection margin was classified as $R_{0}$ in the absence of tumour cells in the resection margins, $R_{1}$ when tumour cells were detected less than $1 \mathrm{~mm}$ from resection margin microscopically and $R_{2}$ in the presence of macroscopic tumour.

Statistical analysis was done using SPSS version 19. Continuous variables were expressed as means and standard deviations. Categorical variables were expressed as counts and percentages. Statistical significance was calculated using Chi-square test, Fisher exact test and Student-t test where appropriate. "P" valve less than 0.05 were considered significant.

\section{Results}

Based on the availability of complete data set, a total of 45 patients were included in the study. Aberrant hepatic artery was present in 11 (24\%) patients with type $V$ being the most common. There was no difference in the age, gender distribution and ASA level of patients in the two groups, as shown in in table 1. Mean operating time (459 vs. 451 minutes) and estimated blood loss (922 vs. 705 $\mathrm{ml}$ ) were higher in group $\mathrm{A}$, but the results were not statistically significant. Number of packed red blood cells transfusions were significantly higher in group A ( $p<$ 0.025), as shown in table 2.

Overall morbidity and 30-day mortality rates were comparable in the two groups, as shown in table 2. One patient in group A died of septic shock and another patient underwent re-exploration for bleeding from aberrant right hepatic artery after failed angioembolization and
Table-1: Demographic Details.

\begin{tabular}{lccc}
\hline Variable & Group A & Group B & p-value \\
\hline Number & $11(24 \%)$ & $34(76 \%)$ & \\
Gender (Male / Female) & $7 / 4(64 \% / 36 \%)$ & $22 / 12(65 \% / 35 \%)$ & 1.00 \\
Age (Years) & $55.36 \pm 13.01$ & $58.44 \pm 8.92$ & 0.38 \\
ASA level (II/III) & $6 / 5$ & $8 / 26$ & 0.071 \\
Hiatt's Classification * & & & \\
Type I & 0 & $34(100 \%)$ & \\
Type II & $2(18 \%)$ & & \\
Type III & $2(18 \%)$ & & \\
Type IV & $2(18 \%)$ & & \\
Type V & $5(46 \%)$ & & \\
\hline
\end{tabular}

ASA: American Society of Anesthesiology, ${ }^{*}$ Reference 3.

Table-2: Comparison of Operative and Outcome Variables.

\begin{tabular}{lccc}
\hline Operative Variables & Group A (n=11) & Group B (n=34) & p-value \\
\hline Operating time (minutes) & $459 \pm 60$ & $451 \pm 66$ & 0.709 \\
Estimated Blood Loss (ml) & $922 \pm 462$ & $705 \pm 468$ & 0.186 \\
PRBC transfused (units) & $1.64 \pm 1.02$ & $0.97 \pm 0.75$ & 0.025 \\
FFPs transfused (units) & $3 \pm 2$ & $2 \pm 2$ & 0.219 \\
Type of resection & & & 0.337 \\
RO Resection & $8(73 \%)$ & $30(88 \%)$ & \\
R1 Resection & $3(27 \%)$ & $4(12 \%)$ & \\
Outcome Variables & & & \\
Morbidity & $9(82 \%)$ & $21(62 \%)$ & 0.288 \\
Clavien-Dindo Classification** & & & \\
Type I & $3(33 \%)$ & $4(19 \%)$ & \\
Type II & $2(22 \%)$ & $7(33 \%)$ & \\
Type III & $2(22 \%)$ & $6(29 \%)$ & \\
Type IV & 0 & $1(5 \%)$ & \\
Type V & $2(22 \%)$ & $3(14 \%)$ & \\
Mortality & $2(18 \%)$ & $3(9 \%)$ & 0.582 \\
Stage of the Disease & & & \\
Stage IA & 0 & $2(6 \%)$ & \\
Stage IB & $1(9 \%)$ & $5(15 \%)$ & \\
Stage IIA & $2(18 \%)$ & $9(26 \%)$ & \\
Stage IIB & $6(55 \%)$ & $15(44 \%)$ & \\
Stage III & $2(18 \%)$ & $3(9 \%)$ & \\
Perineural invasion & $8(73 \%)$ & $14(41 \%)$ & 0.091 \\
Perivascular invasion & $5(45 \%)$ & $11(32 \%)$ & 0.483 \\
Length of Stay (days) & $16 \pm 7$ & $15 \pm 5$ & 0.384 \\
Duration of follow up & $397 \pm 667$ & $516 \pm 523$ & 0.545 \\
\hline PRBC: Packed & &
\end{tabular}

PRBC: Packed red blood cells, FFP: Fresh frozen plasma, ${ }^{*}$ Reference 8.

eventually expired due to multi-organ failure. Two patients in group B died of septic shock secondary to anastomotic leak and hospital acquired pneumonia and the third patient was re-explored and underwent total pancreatectomy due to bleeding from pancreatic bed and eventually expired in intensive care unit.

There was no difference in the oncological clearance in both the groups with $\mathrm{R}_{0}$ resection in $73 \%$ patients in group $A$ and $88 \%$ patients in group $B(p=0.337)$. Majority of 
patients in both groups had stage II disease, with comparable pathologic profile.

\section{Discussion}

Anomalies in the anatomy of hepatic artery is a common entity and found in about $20 \%$ to $40 \%$ of patients undergoing PD. 1 The most common anomaly reported in literature is right hepatic artery arising from SMA in 13\% to $26 \%$ patients. ${ }^{7}$ In our series, $24 \%$ of patients who underwent PD had anomalous hepatic artery anatomy and the most common variant was type $V$ in $46 \%$ patients according to Hiatt's classification in which common hepatic artery (CHA) arises from the Superior Mesenteric Artery (SMA). CHA arising from SMA cross behind or through the head of pancreas before skirting laterally or behind portal vein and penetrates the hepaticoduodenal ligament. It is important for surgeons to be aware of various arterial anomalies in order to avoid injury to this vessel during PD. Inadvertent injury to this vessel can potentially compromise the blood supply of the liver causing hepatic ischaemia as well as ischaemic injury to common bile duct resulting in biliary enteric anastomotic site strictures or fistulas. ${ }^{9}$ None of our patients in this group developed such complications at the follow up duration of one year.

Another surgical consideration in the presence of such arterial anomaly is that an attempt to preserve such vessels can compromise the oncological clearance and radicality of tumour resection. Such efforts can result in positive microscopic $\left(R_{1}\right)$ or macroscopic $\left(R_{2}\right)$ retro-pancreatic resection margin. In our study $73 \%$ patients in group $A$ and $88 \%$ patients in group $B$ had $R_{0}$ resection and there was no significant difference in the oncological tumour clearance in both groups. Our results are comparable to previous similar studies published by Rubio-ManzanaresDorado $\mathrm{M}$ et al ${ }^{10}$ and Sulpice $L$ et al. ${ }^{11}$ The percentage of $R_{1}$ resection in group $A$ was higher $(27 \%)$ in group $A$ as compared to group $B(12 \%)$ which could be secondary to more patients with stage IIB and stage III disease in group A.

Classically, the presence of anatomic variation of hepatic artery used to be a relative contraindication for a PD. ${ }^{3}$ Technical challenges faced during an already difficult procedure can contribute to major surgical implications. Technical difficulties can prolong the operating time and increase the amount of blood loss thus increasing the need for more blood transfusions and post-operative complications. In our present study the operating time, amount of blood loss and need for packed red blood cell and fresh frozen plasma transfusion was higher in group A as shown previously by Rubio-Manzanares-Dorado $M$ et al, ${ }^{10}$ but the difference was only significant in terms of packed red blood cell transfusions. Transfusion rate usually varies depending on the individual anaesthetist's practice at our institution and reflects more difficult dissection of tumour in group ' $A$ ' resulting in more blood loss requiring blood transfusions. Aberrant arterial anatomy seemed to have no influence on the postoperative morbidity and mortality in the current study, which was also shown in various previous studies. ${ }^{10,11}$ Both groups had no statistically significant different complications in postoperative period according to Clevien-Dindo classification. 8

We can conclude that Pancreatoduodencetomy is feasible in patients with aberrant hepatic artery as it does not seem to influence the morbidity, mortality and tumour resection margins in patients undergoing Whipple's procedure. The major limitation of our study is the small number of patients, but this was due to the lack of availability of complete perioperative data set in patients.

Disclaimer: None to declare.

Conflict of Interest: None to declare.

Funding Sources: None to declare.

\section{References}

1. Yang SH, Yin YH, Jang JY, Lee SE, Chung JW, Suh KS, et al. Assessment of hepatic arterial anatomy in keeping with preservation of the vasculature while performing pancreatoduodenectomy: an opinion. World J Surg 2007; 31: 2384-91.

2. Michels NA. Newer anatomy of the liver and its variant blood supply and collateral circulation. Am J Surg 1966; 112: 337-47.

3. Hiatt JR, Gabbay J, Busuttil RW. Surgical anatomy of the hepatic arteries in 1000 cases. Ann Surg1994; 220: 50-2.

4. Saeed M, Murshid KR, Rufai AA, Elsayed SE, Sadiq MS. Coexistence of multiple anomalies in the celiac mesenteric arterial system. Clin Anat 2003; 16: 30-6.

5. Kim PT, Temple S, Atenafu EG, Cleary SP, Moulton CA, McGilvray ID, et al. Aberrant right hepatic artery in pancreaticoduodenectomy for adenocarcinoma: impact on resectability and postoperative outcomes. HPB (Oxford) 2014; 16: 204-11.

6. Jah A, Jamieson N, Huguet E, Praseedom R. The Implications of the presence of an aberrant right hepatic artery in patients undergoing a pancreaticoduodenectomy. Surg Today 2009; 39: 669-74.

7. Zhang L, Sanagapalli S, Stoita A. Challenges in diagnosis of pancreatic cancer. World J Gastroenterol 2018; 24: 2047-60

8. Bolliger M, Kroehnert JA, Molineus F, Kandioler D, Schindl M, Riss $P$. Experiences with the standardized classification of surgical complications (Clavien-Dindo) in general surgery patients. Eur Surg 2018; 50: 256-61. 
9. Traverso LW, Freeny PC. Pancreaticoduodenectomy. The importance of preserving hepatic blood flow to prevent biliary fistula. Am Surg 1989; 55: 421-6.

10. Rubio-Manzanares-Dorado M, Marín-Gómez LM, Aparicio-Sánchez D, Suárez-Artacho G, Bellido C, Álamo JM, et al. Implication of the presence of a variant hepatic artery during the Whipple procedure.
Rev Esp Enferm Dig 2015; 107: 417-22.

11. Sulpice L, Rayar M, Paquet C, Bergeat D, Merdrignac A, Cunin D, et al. Does an aberrant right hepatic artery really influence the shortand long-term results of a pancreaticoduodenectomy for malignant disease? A matched case-controlled study. J Surg Res 2013; 185: 620-5. 